Pathologe 2017 · 38:228

DOI 10.1007/s00292-017-0314-9

Online publiziert: 8. Juni 2017

(c) Springer Medizin Verlag GmbH 2017

CrossMark

\title{
P. Schirmacher
}

Pathologisches Institut, Universitätsklinikum Heidelberg, Heidelberg, Deutschland

\section{Aktueller Stand und Perspektiven der universitären Pathologie}

Fach und damit die Ausbildung des Nachwuchses erfolgt ganz überwiegend an Universitätsinstituten. Längst hat sich auch die Bedeutung der universitären Diagnostik gewandelt. War sie früher geduldetes Mittel zur Sicherstellung von Lehre und Forschung, werden heute alle innovativen diagnostischen Verfahren durch Universitätsinstitute implementiert, validiert und in die breite diagnostische Anwendung „ausgerollt“. Und nicht zuletzt nimmt die interdisziplinäre Integration unseres Faches in den vielen Tumorboards und auch den onkologischen Zentren ihren Ausgang in den Universitätskliniken. Die moderne diagnostische Pathologie ist ohne Wegbereitung durch Universitätspathologien schlicht undenkbar. Der Weg muss dabei für viele Entwicklungen weiter auch zur Verankerung in der nichtuniversitären Pathologie führen. Hierfür brauchen wir eine enge Kooperation zwischen universitären und nichtuniversitären Einrichtungen für Pathologie, wie sie in den letzten Jahren vielfach etabliert wurde und erfolgreich gelebt wird.

Angesichts aller Herausforderungen für unser Fach war es höchste Zeit, die gegenwärtige Situation und Perspektive der universitären Pathologie einer genauen Betrachtung zu unterziehen und Strategien für die Zukunft zu entwickeln. Diese anspruchsvolle und verdienstvolle Aufgabe hat eine Arbeitsgruppe der Deutschen Gesellschaft für Pathologie (DGP) unternommen und präsentiert das komprimierte Ergebnis ihrer Arbeit in diesem Heft. Dafür möchte ich allen Beteiligten einen großen Dank aussprechen. Ich empfehle es allen Pathologen zur Lektüre und hoffe, dass die Arbeit die erhoffte Resonanz im Fach und bei unseren Partnern findet.
Mit den besten Grüßen

Ihr

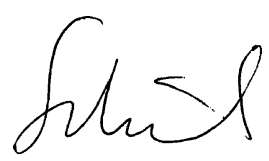

Prof. Dr. P. Schirmacher

\section{Korrespondenzadresse}

Prof. Dr. P. Schirmacher

Pathologisches Institut, Universitätsklinikum Heidelberg

Im Neuenheimer Feld 224, 69120 Heidelberg, Deutschland

Peter.Schirmacher@med.uni-heidelberg.de

Interessenkonflikt. P. Schirmacher gibt an, dass kein Interessenkonflikt besteht. 